\title{
A Comparative Evaluation of Adaptive and Non-adaptive Sliding Mode, LQR \& PID Control for Platform Stabilization*
}

\author{
Emre Akgul ${ }^{1}$, Mehmet Mutlu $^{2}$, Afsar Saranli $^{3}$ and Yigit Yazicioglu ${ }^{4}$
}

\begin{abstract}
During the uniform locomotion of compliant legged robots and other terrain vehicles, the body of the robot often exhibits complex oscillations which may have a disturbing effect on onboard sensors. For a camera mounted on such a robot, due to perspective projection, the effects of angular disturbances are particularly pronounced as compared to translational disturbances. This paper is motivated by the particular problem of legged robots exhibiting angular body motions and attempts to evaluate the performance of baseline and stateof-the-art controllers for compensating this undesired motion. For this comparative evaluation, a simplified planar camera platform is considered in a Matlab-Simulink based simulation environment but motion disturbances are collected on a physical experimental robot platform. Although the full stabilization problem is in 3D with three independent axes of rotation, we currently consider a planar case on the pitch axis with a kinematic structure very similar to many parallel actuated 3D platforms. We believe that despite the simplified analysis, the presented performance evaluation provides significant insight into the general problem. The work consist of the derivation of the planar platform model followed by the implementation and comparative testing of 4 different controllers, namely Proportional-Integral-Derivative (PID), Linear Quadratic Regulator (LQR), Sliding Mode (SMC) and Adaptive Sliding Mode (ASMC) controllers. Experimental setup, disturbance collection and finally, the controller performance test results are presented and discussed.
\end{abstract}

\section{INTRODUCTION}

Vehicles moving on natural terrain, including outdoor mobile robots, are often exposed to a variety of surface conditions. The surface causes a wide frequency band motion disturbance on the vehicle that can range from quasi-periodic low frequency oscillations to high-frequency vibrations. These motion disturbances, depending on the frequency, can have different effects on the vehicle and/or on sensors mounted on the vehicle. To suppress undesired vibrations, in particular on the high-frequency bands, mechanical passive vibration absorbers in the form of spring-damper systems are widely used as low pass filters [1].

Such passive vibration compensation may not be sufficient for applications where precision sensors such as cameras are used on the vehicle. Mobile robotic applications is a typical example. Other application examples are where precise lineof-sight (LOS) positioning of a payload such as a long range

\footnotetext{
*This work is supported by TUBITAK.

${ }^{1}$ Emre Akgul, ${ }^{2}$ Mehmet Mutlu and ${ }^{3}$ Afsar Saranli are with the Department of Electrical and Electronics Engineering, Middle East Technical University, Ankara, 06800, Turkey emrea at eee.metu.edu.tr, mehmet.mutlu at ieee.org, afsars at metu.edu.tr

${ }^{4}$ Yigit Yazicioglu is is with the Department of Mechanical Engineering, Middle East Technical University, Ankara, 06800, Turkey yigit at metu.edu.tr
}

vision system, a satellite dish or a gun turret is required [2]. For all these applications, active control is required, often in combination with passive mechanical filtering, to fully compensate for the effect of these vehicle-ground interaction generated disturbances.

For the case of a camera mounted on the vehicle or mobile robot, the motion disturbances seriously degrade the quality of the acquired vision data, resulting in particular, a degradation known as motion-blur [3]. Motion-blur in turn, is known to degrade the performance of all image feature detectors used in image processing and computer vision. One can use a passive low-pass filter to eliminate vibrations in the high-frequency band, while using an active controller to compensate for the disturbances in the lowfrequency band. In the present study, disturbances from the locomotion of a compliant legged hexapod robot are considered. Hence, the compliant legs constitute the passive part of the filtering. Hence, the study focuses on the active compensation, assuming that the very high-frequency part of the disturbance is attenuated.

Active motion control has to be combined with an appropriate kinematic structure supporting a platform to stabilize payload or sensors. In the 3D general case, these take the form of either Steward platforms [4] (capable of both 3axes translational and 3-axes rotational motion) or gimbaled structures [5] (capable of 3-axes rotational motion). In the simplified planar case considered in the present work, we focus on angular compensation and consider a platform consisting of a rotary joint driven by a brushless linear motor actuated prismatic joint Fig. 1 .

There is a rich body of literature on feedback controllers that one can consider for controlling the nonlinear system resulting from the aforementioned kinematic arrangement. The most basic approach is with a reasonably tuned PID controller [6]. Linear-Quadratic-Regulator (LQR) is a high performance optimal state-space controller. It is nevertheless a linear controller that uses a linearized approximation of the nonlinear plant in its design [7]. In the present study, we consider both of these linear controllers as baselines of performance. We also consider in our study, two nonlinear controller structures, namely Sliding Mode Control (SMC)[8] and Adaptive Sliding Mode Control [9] to comparatively evaluate their performance on this nonlinear problem subject to realistic disturbances.

The motivation of the study is to eventually compensate for the rotational motion induced camera motion-blur on our experimental robotic platform SensoRHex. This is a compliant legged hexapod robot having the proven RHex 
morphology [10] but with improved sensor, actuator and computational infrastructure.

Rest of the paper is organized as follows: Section II gives the dynamic modeling of proposed 1DOF mechanism. Section III discusses the implemented controllers. Section IV comparatively illustrates experimental results obtained by implemented controllers. Conclusions are presented in Section V.

\section{DYNAMIC MODEL}

Main objective of this research is to implement a robot head that can reject the disturbances occurring due to the motion of six-legged robot SensoRHex. During our experiments rotational movements in 3 axis, that could effect the performance of vision based algorithms, are observed. However, in order to begin with a one axis planar head model or in other words a planar head stabilization platform is designed to see and overcome the difficulties of the main problem in a simpler architecture. The model Fig. 1 has the capability of stabilize rotational movements in one direction only with a linear dc motor.

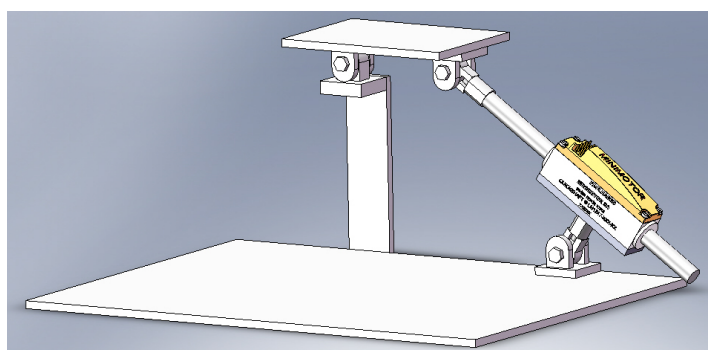

Fig. 1. 3D Solidworks Schematic of Linear Planar Head Model.

Mathematical model of the linear planar head model is derived in order to create a realistic simulation environment. Before starting to model the structure of head stabilization model, parameters of the system is defined.

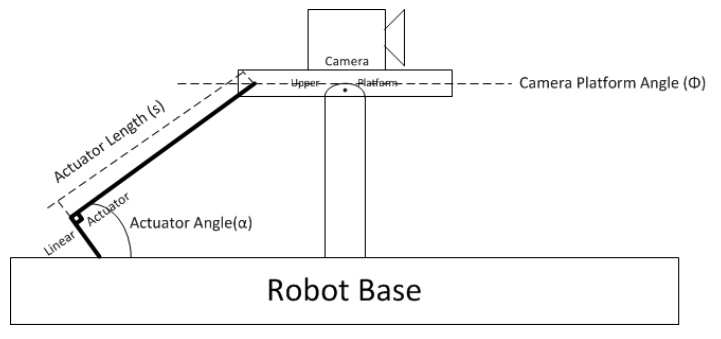

Fig. 2. 2D Schematic of Linear Planar Head Model.

\section{A. Kinematic Analysis}

Before analyzing the system the correspondence of the parameters to the real world is discussed. Fig. 2 shows those parameters in the diagram of head model. $s$ is the length of the linear dc motors shaft. And it is the parameter that will be controlled by control algorithms in order to obtain a stable head movement. More importantly $\phi$ which is the camera platform angle with respect to base platform, that is observed and measured. Moreover $\phi$ or $\dot{\phi}$ are the system variables that will be stabilized by certain control algorithms. Three main position equations are derived in order to aid the dynamic analysis of the system. As Fig. 3 illustrates, $a$ is the height of the stabilization platform, where distance between the end of the actuator shaft and center of the camera platform is $b$ and, $d$ is the horizontal distance between the actuator joint and the center of the camera platform. $c$ is the vertical distance between the actuator joint and actuator shaft. And $s$ is the length of the actuator as discussed. Using complex plane approach in mechanism analysis following equations are derived.

$$
\begin{gathered}
s e^{j \alpha}-b e^{j(\pi-\phi)}=d+j a, \\
s e^{-j \alpha}-b e^{j(\pi-\phi)}=d-j a,
\end{gathered}
$$

From (1) and (2), the following equation is obtained;

$$
s=f(\phi)=\sqrt{a^{2}+b^{2}+d^{2}-c^{2}+2 b d \cos (\phi)+2 b a \sin (\phi)} .
$$

Then by using trigonometric relations;

$$
A \cos (\alpha)-B \sin (\alpha)=C=R \cos (\alpha+\gamma)
$$

$$
R^{2}=A^{2}+B^{2}
$$

$$
\gamma=\tan ^{-1}(B / A)
$$

the required position analysis is obtained as;

$$
\begin{aligned}
& \phi=g(s)=\cos ^{-1}\left(\frac{s^{2}-a^{2}-b^{2}-d^{2}+c^{2}}{\sqrt{(2 b d)^{2}+(2 b a)^{2}}}\right)+\tan ^{-1}\left(\frac{a}{d}\right), \\
& \alpha=h(s)=\cos ^{-1}\left(\frac{s^{2}+a^{2}-b^{2}+d^{2}}{\sqrt{(2 b d)^{2}+(2 b a)^{2}}}\right)+\tan ^{-1}\left(\frac{a}{d}\right) .
\end{aligned}
$$

However in dynamic analysis,

$$
\alpha(t)=j(\phi(t))
$$

is required which is a derivation of the above analysis.

After deriving above equations, speed and acceleration analysis is done in order to complete dynamic analysis for a realistic simulation environment. 


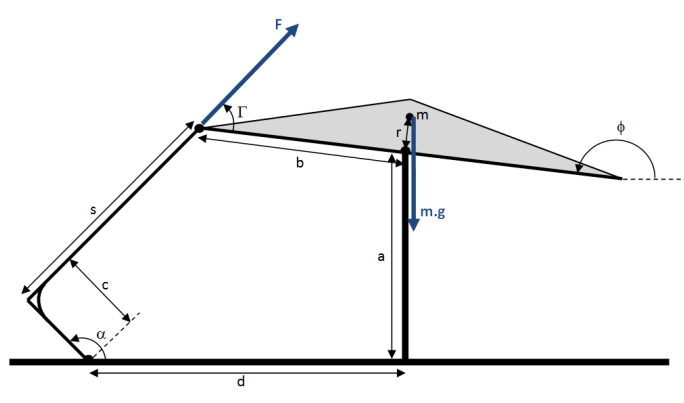

Fig. 3. Diagram of the Planar Head Model.

\section{B. Dynamic Analysis}

Dynamic analysis is required in order to simulate the environment. As seen in Fig. 3, force is applied to the camera platform from the linear actuator which is the control input of the system.

Sum of the all moments around a certain point and all forces in each three axes must be zero. Note that the aim of this analysis is to find the second order equation of $\phi$ as derived in,

$$
\ddot{\phi}(t)=\left[m_{\text {cam }} \operatorname{grsin}(\phi(t))-B_{\text {head }} \dot{\phi(t)}+F(t) \operatorname{asin}(\Gamma(t))\right] \Psi .
$$

In (10) $\Psi$ is given as;

$$
\Psi=\frac{r}{m_{c a m} r+I_{c a m}} .
$$

$\Gamma(t)$ is the angle of force applied to the head stabilization platform and can be written in terms of $\alpha$ and $\phi$ as;

$$
\Gamma(t)=\alpha(t)+90-\phi(t) .
$$

Furthermore, $B_{\text {head }}$ is the viscous friction at the joint which connects the stabilization platform to the body of the robot. $r$ is the distance between the center of mass of the camera. $m_{\text {cam }}$ and $I_{\text {cam }}$ is the mass and the moment of inertia of the camera which will be mounted of the top of the stabilization platform.

In order to create a realistic simulation of the dynamic head stabilization platform, motor model is added. Motor voltage will be the controller output in the real applications, so the relation between the applied force and the motor voltage is derived and added to the model of the system. By inserting;

$$
F(t)=\frac{V_{m}(t)-K_{b} \dot{s}}{R_{a}} K_{t},
$$

to (10) a more realistic simulation model is created. Where $V_{m}(t)$ is the motor voltage, $K_{t}$ is the force constant, $R_{a}$ is the armature resistance and $K_{b}$ is the back emf constant of the motor. Also note that motor inductance is neglected in this formula in order to create a fast working simulation model.

\section{CONTROLlers}

Control of a single actuator may be perceived as a trivial task. However, many requirements such as settling time, overshoot percentage and steady state error should be met at the same time even though the controlled actuator is subjected to large disturbances. Control efforts examined in this paper tries to eliminate rotational disturbances occurred on SensoRHex caused in the pitch axis while SensoRHex walks through a straight line on a concrete surface and keep the angular velocity at zero.

In order to come up with an efficient controller, 4 different types of controllers, PID, LQR, sliding mode controller(SMC) and adaptive sliding mode controller(ASMC), are tested on the platform. And their performances are demonstrated in a comparative evaluation.

\section{A. Proportional Integral Derivative (PID) Controller}

One of the most common controllers used in the literature and industry is PID controller because of its simple structure and efficiency in many applications. First PID controller is used to control the platform.

PID control basically takes the amount of error in the control parameters (angle in our case) and applies input to the system according to its parameters. $K_{P}$ :position constant , $K_{D}$ :derivative constant and $K_{I}$ :integral constant are the three parameters of this control. The $K_{P}, K_{D}$ and $K_{I}$ parameters of PID controller are tuned with known methods [11].

\section{B. Linear Quadratic Regulator ( $L Q R)$}

LQR is an optimal controller that requires state space linear approximation of the non-linear system. LQR generates the plant input as a function of all state variables. State space equations of linear time invariant system is as follows;

$$
\dot{x}(t)=A x(t)+B u(t) .
$$

Also performance index of $\mathrm{LQR}$ controller is introduced as;

$$
J=1 / 2 \int_{0}^{\infty}[x(t) Q x(t)+u(t) R u(t)] d t,
$$

where $u(t)$ is the input and $x(t)$ is the state of the system.

$J$ must be minimal in order to achieve an optimal control. $Q$ and $R$ denote the weighting matrix of the state variable and input variable. An optimal control is dependent on $Q$ and $R$ matrix. However there is no common method in tuning those parameters. Usually simulation trial and error method is used for arranging the correct parameters. Note that in order to implement an optimal control an optimal control input $u(t)$ found . By solving the (15) for minimum cost $u(t)$ could be found as;

$$
u(t)=-K x(t) .
$$

Also $K$ is determined by solving Riccati Matrix Equations. 


\section{Sliding Mode Control (SMC)}

SMC is widely used in several systems, such as nonlinear, multi-input/multi-output, large-scale and infinite-dimensional and stochastic systems. The main advantage of SMC is robustness. A system which is insensitive to parametric uncertainty and external disturbances is possible with SMC. Robot, motor, aircraft, spacecraft and flexible space structure control are some application areas of sliding mode control.

The first step of implementing a SMC is to derive the system equations [8] in the form ,

$$
\ddot{x}(t)=\hat{a} x(t)+\hat{b} \dot{x}(t)+\hat{c} u(t)+\hat{d} .
$$

Which is derived in Section II in details.

$\hat{a}$ and $\hat{b}$ is the system characteristic, $\hat{c}$ is the controller gain that are derived from the second order equation of motion which is given in Section II. And $\hat{d}$ is the disturbance. And the control input is divided into two parts as,

$$
u=u_{\text {cont }}+u_{\text {discont }} .
$$

$u_{\text {discont }}$ is the controller function, which forces the system to the sliding hyper-surface is defined as,

$$
s=\left(\frac{d}{d t}+\lambda\right) \tilde{x},
$$

and $u_{\text {cont }}$ the control function which moves the system to the equilibrium on the sliding surface.

$$
u_{\text {cont }}=\frac{\ddot{x_{d}}-\lambda \dot{\tilde{x}}(t)-\hat{a} x(t)-\hat{b} \dot{x}(t)}{\hat{c}},
$$

gives the formulation of $u_{\text {cont }}$ and,

$$
u_{\text {discont }}=-k \operatorname{sgn}(s) \text {, }
$$

explains $u_{\text {discont }}$.

\section{Adaptive Sliding Mode Control (ASMC)}

Adaptive Sliding Mode Control could be considered as an advanced version of basic sliding mode control that is also in the topics of many advanced control studies. However due to its complicated nature, this method is often used in systems which desires more accuracy and more robustness. By using adaptive sliding mode control, modeling errors, disturbance effects can minimized.

In order to classify a controller as adaptive controller, the controller must adapts itself to the changes of any specific transition. Thus creating a more robust controller by arranging controller parameters accordingly. In order words automatically tuning of controller parameters are achieved by using adaptive approach.

First SMC must be structured, then by making necessary modifications, existing sliding mode controller could be changed into an adaptive sliding mode controller [12]. The control input in the ASMC is given in,

$$
u=u_{\text {cont }}+u_{\text {lin }}+u_{\text {discont }} .
$$

Furthermore for adaptive sliding mode controller case $u_{\text {discount }}$ is changed. Note that adaptive sliding mode controller could be obtained by using many different approaches. However the main idea behind this controller is changing the input force to the system as some parameters of the system changes.

In adaptive sliding mode control approach, $u(t)$ changes as some designer selected parameters change in the system during operation. In this study $u_{\text {discont }}$ is modified as the parameters of the system changes. As seen in,

$$
u_{\text {discont }}=-\left(\frac{1}{c_{o}}|s|+\frac{1}{c_{1}}|s||e|+\frac{1}{c_{2}}|s||\dot{e}|\right) \operatorname{sgn}(s),
$$

the control input is related with the sliding surface $(s)$, error and the rate of change of error. So as those parameters increase a greater control input is applied to the system and $c_{o}, c_{1}$ and $c_{2}$ are the adaptivity parameters. Moreover an additional terms exists as,

$$
u_{\text {lin }}=K_{a s m c} s .
$$

\section{EXPERIMENTAL RESUlTS}

\section{A. Experimental Setup}

Simulation results for stabilizing the head movements are illustrated in this section. Results with three different robot velocities are illustrated for all 4 controllers of interest and comparative evaluations are done. Simulation environment is created using Matlab-Simulink.

After obtaining a valid test setup, the performance of the system is tested with using simple signals. However since the main purpose of this study is to obtain a head stabilization platform which is able to stabilize the legged locomotion based disturbances at the robots body, those signals must be obtained. In order to achieve this task an inertial measurement unit (IMU) is used and three different experiments are conducted with three different robot speeds. Angular velocity of the robots body is measured. Experiments are conducted in three different robot locomotion modes namely $M O D E-A$, $M O D E-B$ and $M O D E-C$. There is no strict linear relation exists among them due to the morphology of the six-legged robot SensRHex.And those modes have corresponding linear velocities as; $13 \mathrm{~cm} / \mathrm{s}, 18 \mathrm{~cm} / \mathrm{s}$ and $44 \mathrm{~cm} / \mathrm{s}$ respectively.

Then the measured IMU signals are given to the controllers as a reference signal and the tracking ability of the controllers are measured in terms of performance measure which is the squared sum of all error values measured at discrete time intervals thorough out the experiment time. The main idea behind this action is, if a system is able to track a disturbance signal then it could easily stabilize the system.

As illustrated in Section I during locomotion of SensoRHex, robot's body is exposed to oscillations. However, those oscillations have high frequency components. So initially a passive isolation is applied to the system which acts like a low pass filter. The real life equivalent of this filter is a simple spring damper structure. 


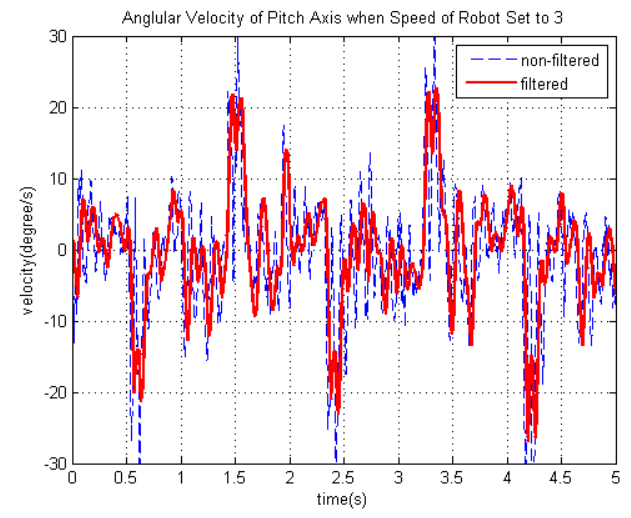

Fig. 4. Low-pass Filtered and Non Filtered Angular Velocity of Robot Body at Robot Locomotion is Set to MODE A.

After obtaining passive isolation, the rest of the oscillations are tried to be canceled out by active control with the designed head stabilization platform.

\section{B. Performance of Baseline Controllers with Realistic Dis- turbances}

Performance of the head stabilization platform is tested by using baseline controllers like PID and LQR. Simulation results for different velocities are illustrated in Fig. 5, Fig. 6 and Fig. 7. Moreover the performance measure of the system is chosen as the squared sum of all error values measured at discrete time intervals. And TABLE I shows the numeric results of the PID and LQR controller for different robot locomotion modes.

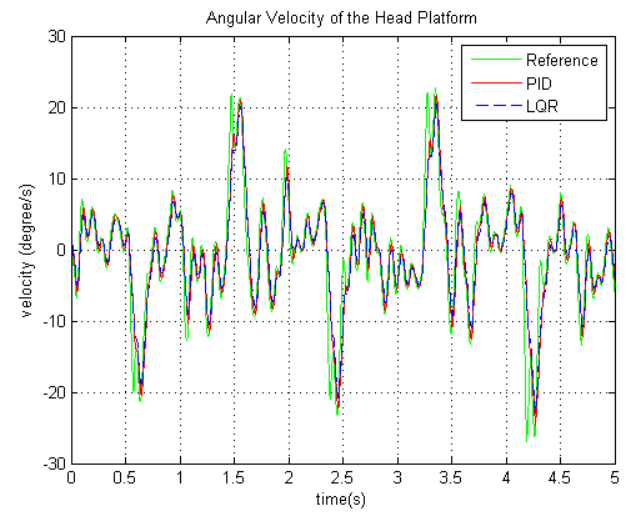

Fig. 5. Angular Velocity of Robot Body at Robot Locomotion is Set to MODE-A and its Tracking With PID and LQR Controllers.

Those two controllers are the most common controllers in the control application area. So initially their performances are obtained in order to create a relevant comparison base.

\section{Performance of Adaptive and Non-adaptive Sliding Mode Control with Realistic Disturbances}

After observing the performance of above baseline controllers, non-linear and robust controllers are tried on the

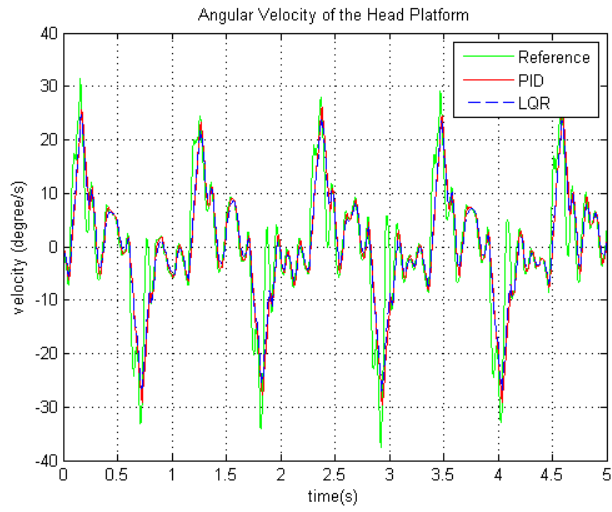

Fig. 6. Angular Velocity of Robot Body at Robot Locomotion is Set to MODE-B and its Tracking With PID and LQR Controllers.

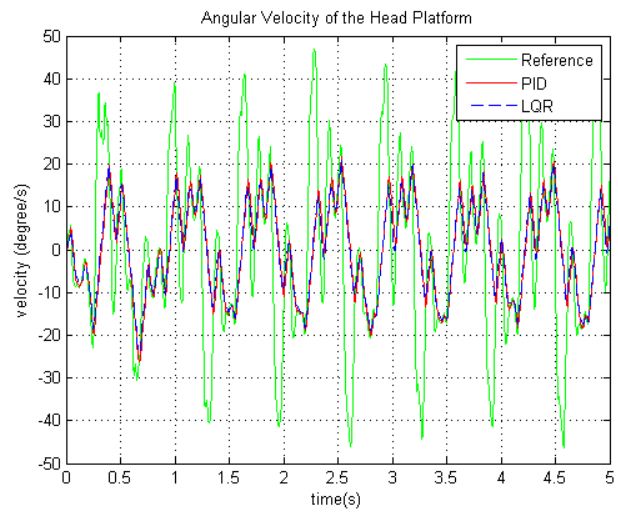

Fig. 7. Angular Velocity of Robot Body at Robot Locomotion is Set to MODE-C and its Tracking With PID and LQR Controllers.

head stabilization platform. Since the head stabilization platform is also a nonlinear system. The performance of those controllers are expected to be higher.

Simulation results of stabilization of robots movements for different robot locomotion modes are illustrated in Fig. 8, Fig. 9 and Fig. 10. And TABLE I shows the numeric results of the SMC and ASMC controller for different robot locomotion modes in terms of performance measures.

From those results the stabilization ability of SMC and ASMC on our platform is clearly observed.

PID, LQR, Sliding Mode and Adaptive Sliding Mode controllers are studied for camera/head stabilization platform control. Using an Adaptive Sliding Mode Controller instead of baseline controllers and SMC results in more robust control over the wider range of a frequency band for our stabilization platform. TABLE I illustrates the adaptability of ASMC. Residual error of ASMC is 2 times smaller than the residual error of SMC when the robot locomotion is set to $M O D E-A$ and $M O D E-B$. However, when robot speed is set to MODE $-C$, the residual error of ASMC shrinks to one third of the residual error of SMC. Therefore, the adaptability of ASMC is also shown. 


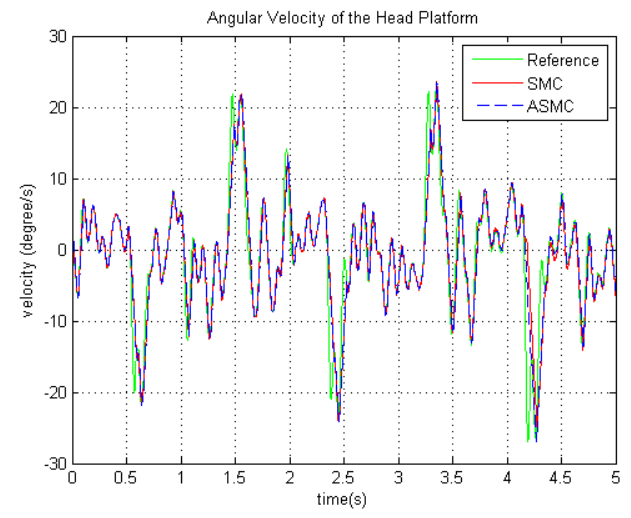

Fig. 8. Angular Velocity of Robot Body at Robot Locomotion is Set to MODE-A and its Tracking With SMC and ASMC.

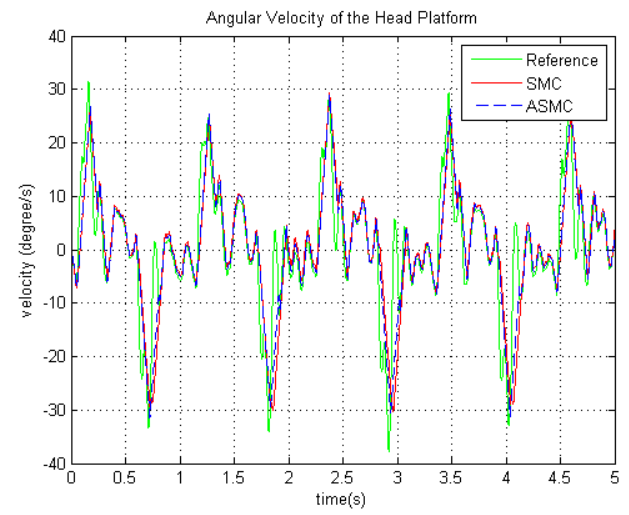

Fig. 9. Angular Velocity of Robot Body at Robot Locomotion is Set to MODE-B and its Tracking With SMC and ASMC.

\section{CONCLUSION}

In this study the performances of PID, LQR, Sliding Mode and Adaptive Sliding Mode controllers are comparatively evaluated on a simplified planar head stabilization platform through Matlab-Simulink simulations. Simulation results are obtained for realistic disturbances obtained from the actual body motion of the robot SensoRHex operated at different locomotion modes. Fair and detailed performance evaluations of all controllers of interest are given. In TABLE I the numerical comparison is given in terms of performance measure which is the sum of the square of all error values measured at discrete time intervals throughout the experiment

TABLE I

Comparison of Controllers In Terms of Performance MEASURE.

\begin{tabular}{|c|c|c|c|}
\hline Controller & MODE-A & MODE-B & MODE-C \\
\hline PID & $3.36 r^{2} / s$ & $8.45 r^{2} / s$ & $97.12 r^{2} / s$ \\
\hline LQR & $2.38 r^{2} / s$ & $9.6 r^{2} / s$ & $106.35 r^{2} / s$ \\
\hline SMC & $0.10 r^{2} / s$ & $0.66 r^{2} / s$ & $14.29 r^{2} / s$ \\
\hline ASMC & $0.05 r^{2} / s$ & $0.32 r^{2} / s$ & $4.39 r^{2} / s$ \\
\hline
\end{tabular}

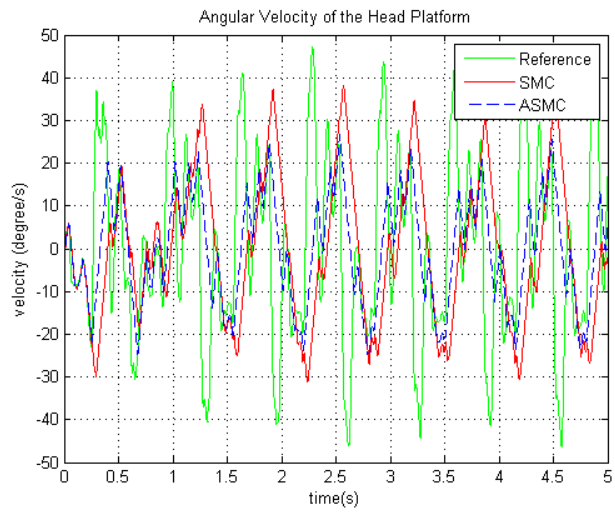

Fig. 10. Angular Velocity of Robot Body at Robot Locomotion is Set to MODE-C and its Tracking With SMC and ASMC.

time. In order to obtain a fair comparison, remarkable effort is given to the theoretical and experimental optimization of parameters used in controllers since the performance of controllers is a function of controller parameters. With parameter set used for controllers: LQR controller performed slightly better than PID controller, ASMC performed more robust to velocity changes than SMC due to its adaptation and SMC and ASMC exhibited considerably better performance than both PID and LQR controllers.

\section{ACKNOWLEDGMENT}

Authors would like to thank to all members of their laboratory ROLAB in Electrical and Electronics Engineering in METU.

\section{REFERENCES}

[1] J. Q. Sun, M. R. Jolly, and M. A. Norris, "Passive, adaptive and active tuned vibration absorbers-a survey," Journal of Vibration and Acoustics, vol. 117, no. B, pp. 234-242, 1995.

[2] J. Hilkert, "Inertially stabilized platform technology concepts and principles," Control Systems, IEEE, vol. 28, no. 1, pp. $26-46$, feb. 2008.

[3] S. Nayar and M. Ben-Ezra, "Motion-based motion deblurring," Pattern Analysis and Machine Intelligence, IEEE Transactions on, vol. 26, no. 6, pp. $689-698$, june 2004.

[4] D. Stewart, "A platform with six degrees of freedom," Proc. Inst. Mech. Eng., vol. 180, 1965/1966.

[5] M. Masten, "Inertially stabilized platforms for optical imaging systems," Control Systems, IEEE, vol. 28, no. 1, pp. 47 -64, feb. 2008.

[6] K. Ogata, Modern Control Engineering, 4th ed. Upper Saddle River, NJ, USA: Prentice Hall PTR, 2001.

[7] D. Kirk, Optimal control theory: an introduction, ser. Dover books on engineering. Dover Publications, 2004.

[8] V. Utkin, Sliding Modes and Their Applications in Systems of Variable Structures. Mir, 1978.

[9] E. K. N. Q. Hoang, "A robust adaptive sliding mode controller for remotely operated vehicles," Technische Mechanik, vol. 28(3-4), pp. 185-193, 2008.

[10] U. Saranli, M. Buehler, and D. E. Koditschek, "Rhex: A simple and highly mobile hexapod robot," International Journal of Robotics Research, vol. 20(7), pp. 616-631, 2001.

[11] R. Kelly, "A tuning procedure for stable pid control of robot manipulators." Robotica, vol. 13, pp. 141-148, 1995.

[12] R. Cristi, F. A. Papoulias, and A. J. Healey, "Adaptive sliding mode control of autonomous underwater vehicles in the dive plave," IEEE Journal of Oceanic Engineering, vol. 15(3), pp. 152-160, 1990. 